\title{
Terabit per Second Optical Wireless Links for Virtual Reality Technology
}

\author{
Olivier Bouchet ${ }^{\mathrm{a}}$, Marc Lanoiselée ${ }^{\mathrm{a}}$, Dominic O'Brien ${ }^{\mathrm{b}}$, Ravinder Singh ${ }^{\mathrm{b}}$, Mir Ghoraishi ${ }^{\mathrm{c}}$, Rafael \\ Perez $^{\mathrm{d}}$, Víctor Guerra ${ }^{\mathrm{d}}$, Suat Topsu ${ }^{\mathrm{e}}$ and Jorge Garcia-Marquez ${ }^{\mathrm{e}}$ \\ ${ }^{\mathrm{a} O r a n g e}$ Labs, 4 rue du Clos Courtel, 35512 Cesson Sévigné; ${ }^{\mathrm{b}}$ University of Oxford, Department of \\ Engineering Science, Parks Road, Oxford, OX1 3PJ, UK; ' pureLiFi, 9 Haymarket Terrace, \\ Edinburgh, EH12 5EZ, UK; ${ }^{\mathrm{d} I D e T I C-U L P G C, ~ C / ~ j u a n ~ d e ~ Q u e s a d a ~ 30, ~} 35001$ Las Palmas de Gran \\ Canaria, Spain; 'Oledcomm, 10-12 avenue de l'Europe, Vélizy, 78140, France.
}

\begin{abstract}
The information networks of the future will consist of an all-optical core, with wireless access technologies wherever possible. The fibre networks are extending their reach rapidly, and will further extend to individual spaces within homes and office buildings. The data traffic on networks and the demand for wireless services are also growing exponentially and the nature of services is also evolving with rapid increase in the number of devices. A new generation of 3D displays, with the ability to create Virtual Reality (VR) environments, is being launched. VR technology places significant demands on bandwidth, latency, positioning and mobility.

One challenge addressed by our European collaborative project WORTECS is the development of an optical wireless system able to deliver ultra-high throughput (up to Tbps). The first demonstrator focuses on a high density network that can provide > 1 Gbps per user with multi user, but has the potential to provide Tbps per indoor environment. The second demonstrator focuses on ultra-high data rate links with a novel fibre-optical wireless-fibre approach to create Tbps capable links. VR is targeted as a demanding application, however, other applications include wireless data centers and aircraft cabins.

In this paper, after introduction on the demand for wireless Terabit/s communication, we will focus on VR use case and the need for multi-Gigabit/s data rates. Then we will present the challenges for the project and propose new optical wireless system architecture and system engineering associated to new approach in space and frequency diversity with OFDM and adaptive bit rate for VR.
\end{abstract}

Keywords: Optical Wireless Communication, OWC, Infrared, IR, PLC, OFDM, Virtual Reality

\section{INTRODUCTION}

The information networks of the future should be an all-optical core associated with wireless access technologies wherever possible, and evolution to this model is already underway. The fibre network is already reaching many homes, and will further extend to individual spaces within homes and office buildings. Figure 1 shows the development of Passive Optical Network (PON) architectures, demonstrating that capacities of 100 Gigabit per second (Gbps) will be available by 2019. Increasing this data rate is already ongoing with the use of Wavelength Division Multiplexing (WDM) solution. This will ensure that ultra-high data rate services will be available to citizens whenever they are indoors. 


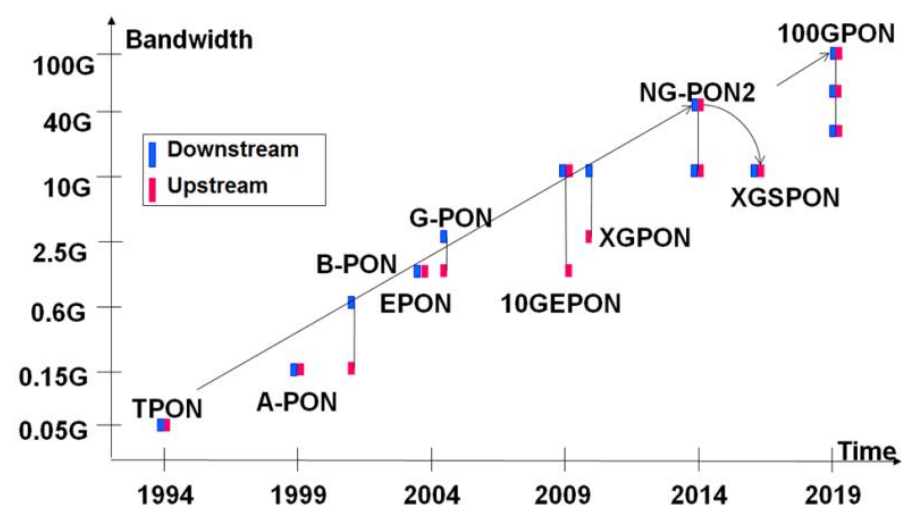

Figure 1. Throughput capacity trend for PON $(\mathrm{G}=\mathrm{Gbps})[1]$.

The traffic and demand for wireless services are also growing exponentially. Much of this traffic will be for indoor data services. The nature of these services is also evolving with the rapid increase in the number of devices and new image based services. A new generation of 3D displays, with the ability to create Virtual Reality (VR) environments, is being launched. VR places significant demands on bandwidth, latency, positioning and mobility, and this will become more challenging as systems evolve and consumers demand of Quality of Experience (QoE) increase.

The wireless infrastructure provides the bridge between the fixed fibre network and the devices that deliver the services to end-users. The key goals for the evolution [2] of the wireless access technology are:

- Extremely high data rate with low latency for Point-to-Point (PtP) and Point-to-Multipoint (PtM) communications (Tbps/space).

- Define solutions to address multiple use (or multiple access) cases in heterogeneous environment.

\section{WORTECS PROJECT PRESENTATION}

The WORTECS project (Wireless Optical \& Radio TErabit CommunicationS), addresses the "Networking research beyond 5G" research topic outlined in the call of the Horizon 2020 (H2020) European Work Programme 20162017 [3]. The scope of the project addresses novel demand-attentive and cooperation networking alternative to 5G in order to combine the wireless approaches to achieve the ultra-high data rates. This will be illustrated on the example use case of Virtual Reality [4].

The primary challenge addressed in the project is the development of a system able to deliver ultra-high throughput (up to Tbps). It will also meet stringent low latency and positioning requirements to address not only the anticipated end-users traffic demands after the 2020 time frame, but also the potential new and currently unknown demands that may arise as a consequence of new ways of using wireless communication networks in the future. Key conceptual elements to be investigated, enabling such low latency and positioning requirements, include innovative network protocol, new signal processing algorithms and design of novel access technologies. Demonstrators built on advanced high performance computing techniques will be developed and used to check link and system level performance in representative environments.

WORTECS consortium addresses these challenging requirements thanks to the experience of its partners. WORTECS brings together several European industrial players (Orange, B-COM and Oledcomm from France, IHP from Germany, pureLiFi from UK) as well as academics (University of Oxford from UK and University of Las Palmas from Spain). Figure 2 shows how WORTECS will meet these capacity demands. The WORTECs concept will emphasize:

- High density Optical Wireless Communications (OWC) and LiFi expertise provided by industry leaders Oledcomm and pureLiFi.

- Ultra-high data rate infra-red expertise links provided by Orange Labs and University of Oxford.

- $\quad$ Ultra-high data rate radio links expertise provided by IHP and B-COM.

- A compelling VR application expertise provided by B-COM.

- Multi-technologies management led by IHP, Orange Labs, University of Las Palmas and B-COM. 


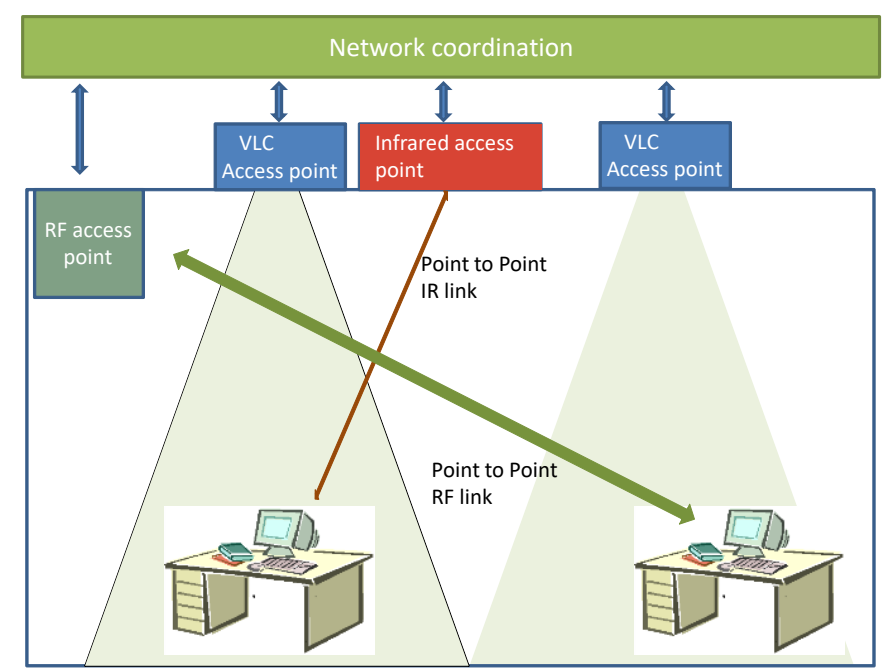

Figure 2. WORTECS wireless access capabilities

\section{SYSTEM DESIGN}

\subsection{System Overview}

For WORTECS demo-1, which targets $1 \mathrm{Gbit} / \mathrm{s}$, we have begun investigations by developing an easy to integrate, compact and cost effective OWC demonstrator, while relaxing the data-rate targets. Our work is based on ACEMIND European collaborative project [5] where we wanted to associate two technologies, Visible Light Communication (VLC) and Power Line Communication (PLC) in order to optimize both infrastructure network and Access Point (AP).

The concept was already introduced [6], whereas the one of the goals of the current project is to take advantage from latest PLC (Power Line Communication) innovations and apply them in the OWC. Indeed, conventional PLC products use two of the three copper wires (Phase $\mathrm{P}$ and Neutral N) to provide power-line Internet. The latest standard HPAV2 offers the possibility to access in addition to Ground $G$ conductor and exploit the physical potential of this electric cable with additional features such as: Time Division Duplexing (TDD) mechanism, MIMO (Multiple Input Multiple Output) capability, OFDM (Orthogonal Frequency Division Multiplexing) modulation and Turbo Convolutional Code (TCC) higher Code Rates (8/9 code rate).

The result is not only significantly a better performance for transmissions, up to $1300 \mathrm{Mbps}$, but also higher range. Our innovation is based on the use of these dual channels in PLC field by the use of two distinct wavelengths in the OWC domain. The additional novelty is to perform the data detection at the receiver side in a differential mode, as already shown in work [7] and thus increase link performance including throughput and distance in OWC domain.

Figure 3 shows the conventional HPAV2 MIMO communication principle with "tp-link" selected PLC plug, AV 1300 Gigabit [8]. 


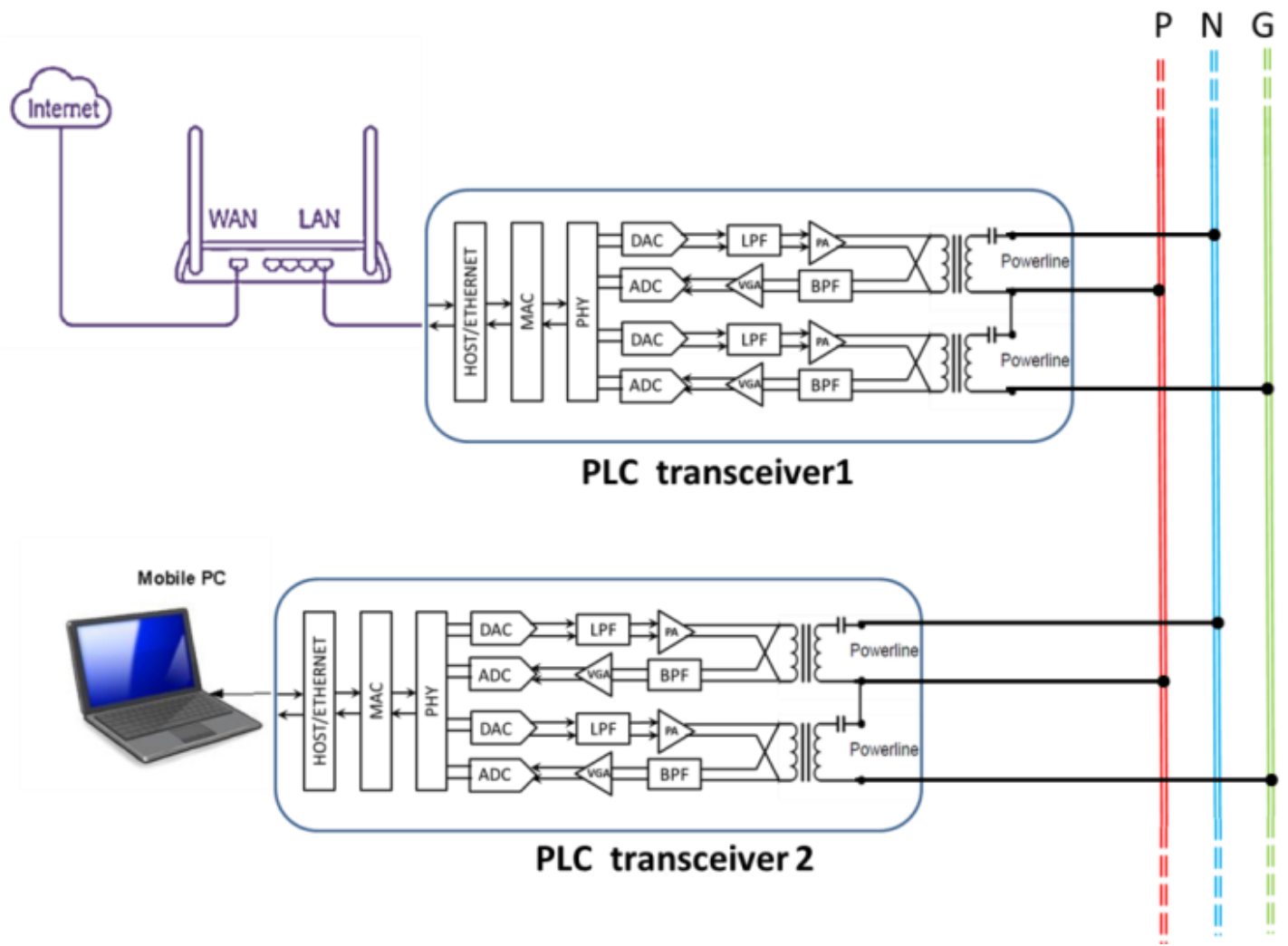

Figure 3. PLC kit "tp-link" - AV 1300 Gigabit

Figure 4 shows the modifications achieved to obtain OWC link from 2 devices. The electrical power coupling module has been removed and replaced by, for each Plug, the following elements. On emitter, we mount Light Emitting Diode (LED) infrared emitter $(850 \mathrm{~nm})$ - SFH 4557 from Osram [9] and LED infrared emitter (950 nm) - SFH 4547 from Osram [10] with Current Amplifier for each LED. On receiver, we assemble Polymethylmethacrylate (PMMA) Hemispherical lenses with $10 \mathrm{~mm}$ diameter associated to an optical bandpass filter with $+/-25$ nanometer (nm) around the central wavelength (850 and 950 respectively); then Positive-Intrinsic-Negative Photodiode (PIN PD) - SFH 2500 from Osram with 800/1000 nm range [11] linked to Complementary Metal-Oxide Semiconductor Trans-Impedance Amplifier (CMOS TIA). The last choice is a good compromise between voltage and noise current face to Bipolar or Junction Field Effect Transistor (JFET) [12] alternatives, 


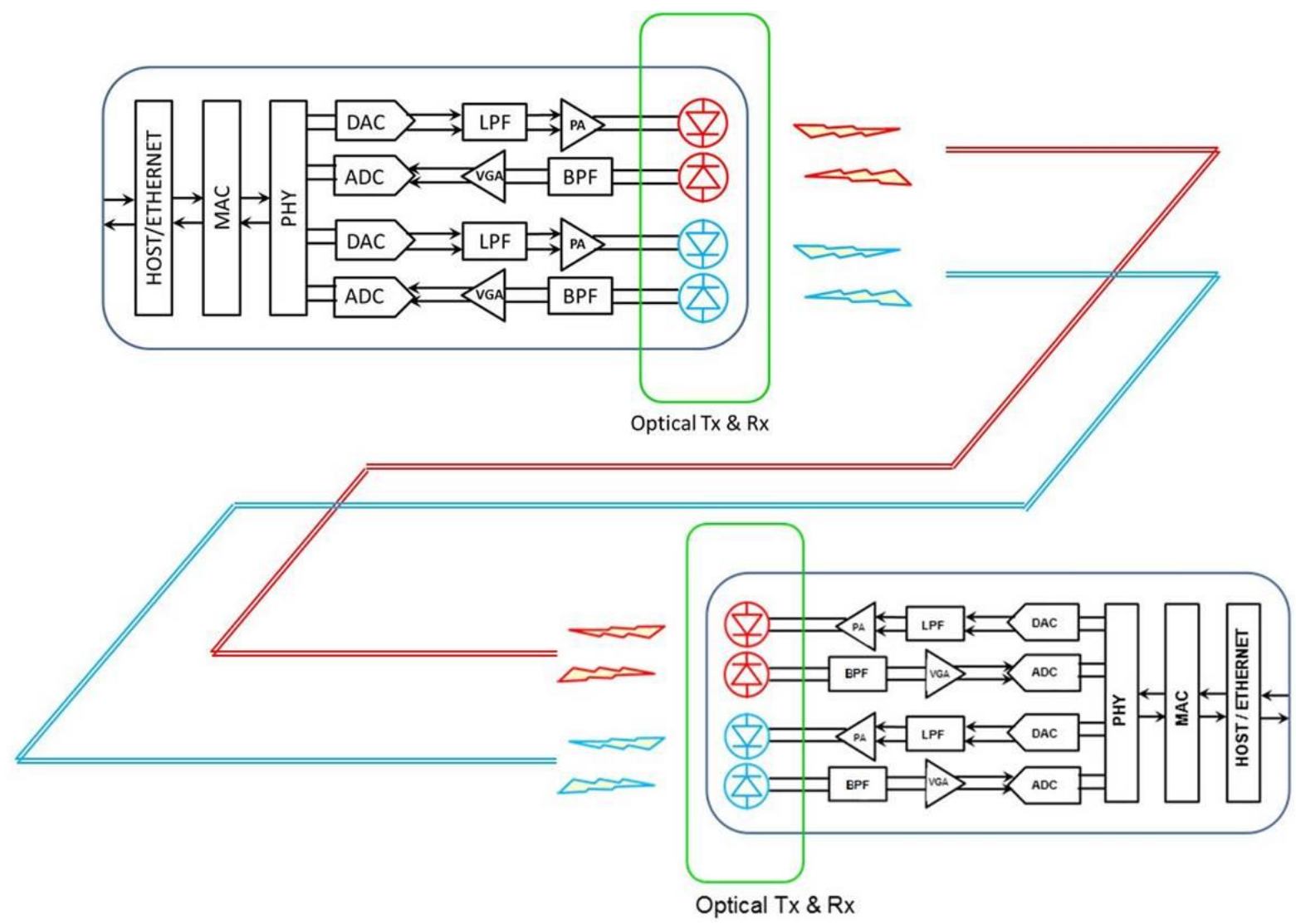

Figure 4. PLC link with optical wireless communication adaptation

Emission is carried out with two wavelengths in differential mode, i.e. one wavelength is modulated by the signal according to one polarity and the other one is modulated with the inverted polarity. At the receiver, the two signals processed by the two photodiodes are therefore of opposite polarities and the subtraction of the second signal by the first one gives the possibility to obtain a global signal with a double amplitude $(+6 \mathrm{~dB})$.

The different noise contributions, that affect the two links and which result mainly from the transmission loss, are statistically independent; the noise level increases by $3 \mathrm{~dB}$. The SNR therefore leads to a gain around $+3 \mathrm{~dB}$. In our configuration, for each wavelength, the signal is modulated and does not need complementary signal to achieve transmission. Differential modulation mode advantage lies by the capacity to suppress or strongly resorb broadband light jammers that could affect transmission.

\subsection{Link Budget}

The prototype is an indoor full-duplex optical wireless communication link with Ethernet interface. The TX (LED)/RX (PIN PD) couple selection was motivated mainly for cost reasons, ease of implementation and especially integration capacity. Indeed, the goal is to maintain volumetric, energy and electrical constraints without having to change the fundamental electronic card characteristics and components on it. These choices have direct consequences on demonstrator functionality and especially on expected performances. For instance, from TX/RX features, it is possible to evaluate the optic link budget [13]. Figure 5 shows a Line Of Sight (LOS) link with the AP installed in the ceiling and the module at a given distance $\mathrm{d}$ and inside AP coverage area. This LOS configuration presents Interferences InterSymbols (ISI) limitation.

Table 1 gives us the optical system parameters. If we want to maintain a positive link budget and a full duplex communication, the maximum distance is 0.25 meter. To increase throughput and distance, a TX (Laser)/RX (PD APD Avalanche Photodiode) couple has a much more relevant potential. Special attention should be paid to the reception sensitivity module. It is a difficult exercise because it does not correspond in any case to the photodiode sensitivity value only. Indeed, it is necessary to take into account the beam concentration losses, optical concentrator and filters losses, 
and especially electronic module amplification, impedance adaptation, filtering, and digital/analog conversion. In our example, we go from $-59 \mathrm{dBm}$ to $-25 \mathrm{dBm}$ for reception sensitivity module and we assume $34 \mathrm{~dB}$ loss. We encountered difficulties to adapt the OWC module with the existing PLC plug electronic board.

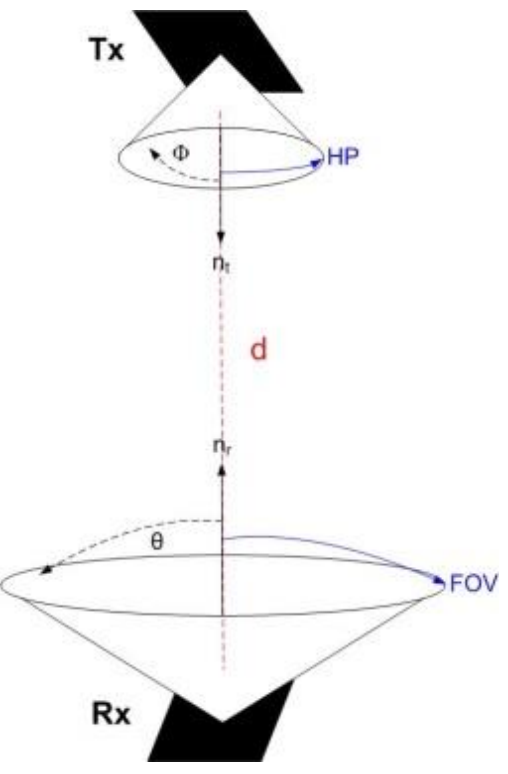

Figure 5. LOS optical link

\begin{tabular}{|c|c|c|}
\hline Wavelength (nm) & $850 \mathrm{~nm}$ & $950 \mathrm{~nm}$ \\
\hline Tx Power - Pt (mW) & 60 & 55 \\
\hline Half Power angle - HP $\left(^{\circ}\right)$ & 30 & 30 \\
\hline Tx angle/normal $n^{t}-\varphi\left(^{\circ}\right)$ & 0 & 0 \\
\hline Rx Sensitivity - Se (dBm) & -25 & -25 \\
\hline Field Of View - FOV $\left(^{\circ}\right)$ & 15 & 15 \\
\hline Area Rx - A $\left(\mathbf{m m}^{2}\right) /$ normal $\mathbf{n}^{r}$ & 4.00 & 4.00 \\
\hline Rx angle/normal $n^{r}-\theta\left({ }^{\circ}\right)$ & 0 & 0 \\
\hline Distance - d (m) & 0.26 & 0.25 \\
\hline m value (Lambert model) & 4.82 & 4.82 \\
\hline n value (Lambert model) & 20 & 20 \\
\hline Tx Power - Pe $/ \varphi(\mathrm{mW})$ & 55.59 & 50.96 \\
\hline Area Rx Efficient - Aeff $\left(\mathrm{mm}^{2}\right) / \theta$ & 4.00 & 4.00 \\
\hline HO & $5.48 \mathrm{E}-05$ & $5.93 \mathrm{E}-05$ \\
\hline Geometric Attenuation - Aff (dB) & -42.61 & -42.27 \\
\hline Power Received - Pr (dBm) & -24.83 & -24.87 \\
\hline Marging Link (dB) & $\mathbf{0 . 1 7}$ & $\mathbf{0 . 1 3}$ \\
\hline Covearge area @ distance $d\left(\mathrm{~m}^{2}\right)$ & $\mathbf{0 . 0 7}$ & 0.07 \\
\hline
\end{tabular}

Table 1. Parameters (grey) and link simulation results

\subsection{Setup}

Figure 6 shows PLC plug "tp-link" - AV 1300 Gigabit modified. The TX module is constituted by 850 and 950 $\mathrm{nm}$ LED. Each RX module is arranged with hemispherical lens, then passband optical filter and PIN photodiode. The global cost for each terminal or plug prototype is around $100 €$. Due to power consumption needs (6 W), it is even possible to use terminal with a battery or plugged to USB port from laptop for instance.

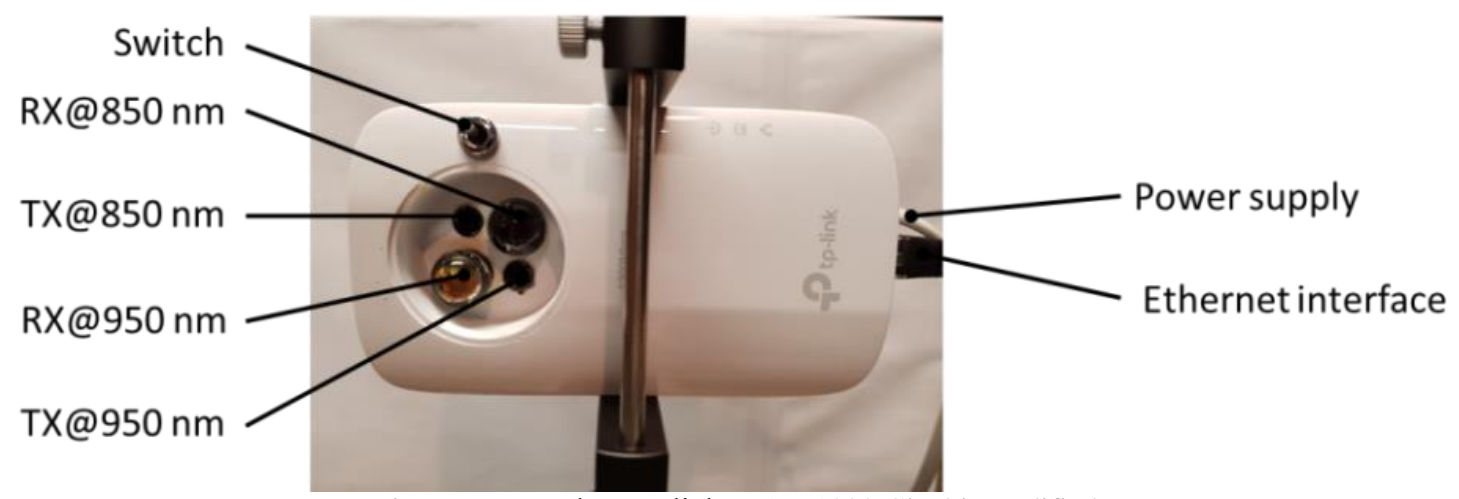

Figure 6. PLC plug “tp-link” - AV 1300 Gigabit modified 


\section{EXPERIMENTAL RESULTS}

\subsection{Testbed presentation}

OWC prototype testbed is shown in Figure $7 \mathrm{a}$ and the link is on Figure $7 \mathrm{~b}$.

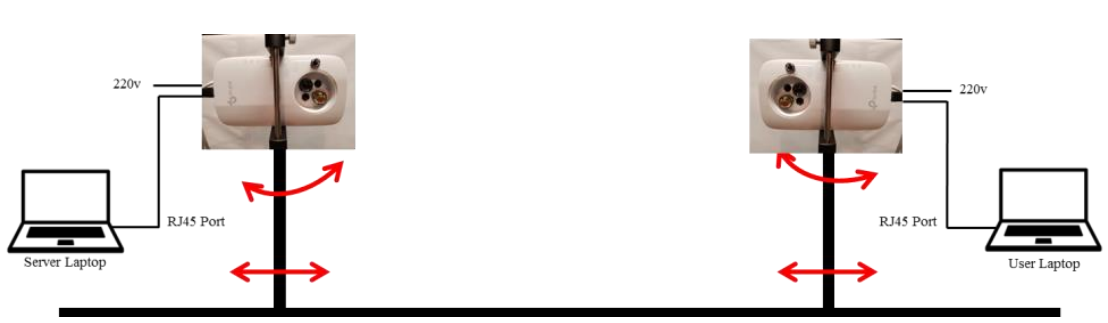

Figure 7a. Testbed

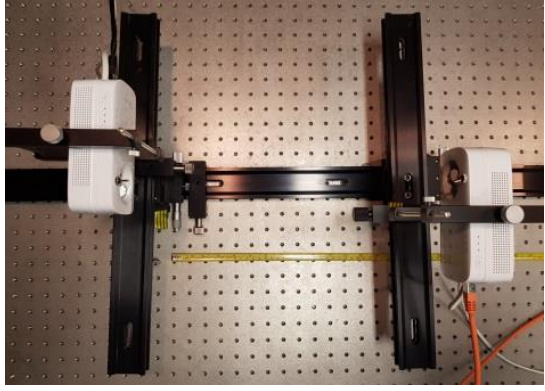

Figure 7b: Complete link

Link measurements were performed with Ethernet network data analyzer (Chariot [14]) which is based on RFC2544 standard. This standard defines specific tests to measure and report network performance. The results give the opportunity to provide a comparable data from different devices and manufacturers. Some tests conducted were also on optic. The tests are: Spectral analysis, throughput, latency and longtime testing.

\begin{tabular}{|l|l|l|l|}
\hline \multicolumn{1}{|c|}{ Functions } & \multicolumn{1}{|c|}{ Brand } & \multicolumn{1}{c|}{ Product } & \multicolumn{1}{c|}{ Features } \\
\hline Optic power Meter & Melles GRIOT & 13 PEM 001 & Thermic - Diam 10mm - Power mini 1 to 3 mW \\
\hline Sensor meter & Melles GRIOT & 13 DPH 101 & $\begin{array}{l}\text { Head measurement Ge - Diam 4.8 mm } \\
800-1800 \mathrm{~nm} \text { 10nW -2mW (Captation area 1 } \mathrm{cm}^{2} \text { ) }\end{array}$ \\
& & Avaspec 2048 & $490-1000 \mathrm{~nm}$ \\
\hline Spectral analyser & Avantes & Latitude E640 & Windows 7 CPU=2.5Ghz, RAM=4Go. Link to Spectral Analyser \\
\hline Laptop & Dell & $\begin{array}{l}\text { Precision } \\
\text { M4400 }\end{array}$ & $\begin{array}{l}\text { Windows XP SP3, CPU=2,8Ghz, RAM=3.5Go } \\
\text { For AP Plug with Software: Chariot v7.30 SP 1 }\end{array}$ \\
\hline Laptop & Dell & EliteBook 850 & Windows 10 64b CPU=2.3Ghz, RAM=8Go. Link to Device Plug \\
\hline Laptop & HP & \multicolumn{2}{|c|}{ Tabe } \\
\hline
\end{tabular}

\subsection{Results}

\section{Spectral analysis}

The spectral analysis was carried out during the link communication for $850 \mathrm{~nm}$ then $950 \mathrm{~nm}$ wavelength. Before the test, a spectral analyzer calibration (black recording and offset for 0 references) was done. During the measurement process, no external light was switched on. The results are (for both plug):

- The central wavelength is equal to $856 \mathrm{~nm}$ with a Full Width at Half Maximum (FWHM) of $26 \mathrm{~nm}$ (Figure 8a).

- $\quad$ The central wavelength is equal to $954 \mathrm{~nm}$ with a Full Width at Half Maximum (FWHM) of $33 \mathrm{~nm}$ (Figure $8 \mathrm{~b}$ ).

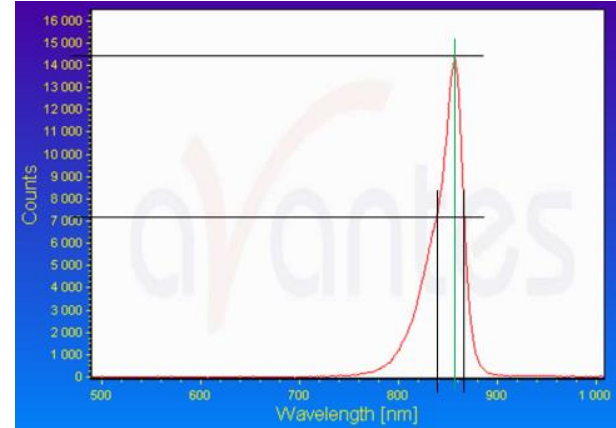

Figure 8a: TX@856 nm with 26 nm FWHM

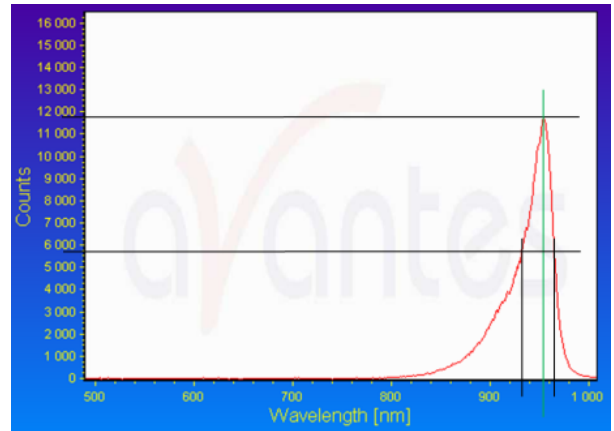

Figure 8b: TX@954 nm with 33 nm FWHM 


\section{Throughput}

The traffic distribution is PtP and there is no Virtual LAN (VLAN) tag. The protocol is Ethernet MAC with IPv4 version. The device type is "Store and Forward ". The test duration is 120 seconds for each distance. There is no target tolerance loss, i.e. frame rate is validated only when no error is computed.

Testbed result is shown on Figure 9. The test process is as following, from a maximum received power level, the signal is gradually attenuated by increasing the distance between the AP Plug and the Plug linked to user laptop via RJ45 port. We measured the optic level power only when there is no communication for $850 \mathrm{~nm}$ and $950 \mathrm{~nm}$ link respectively.

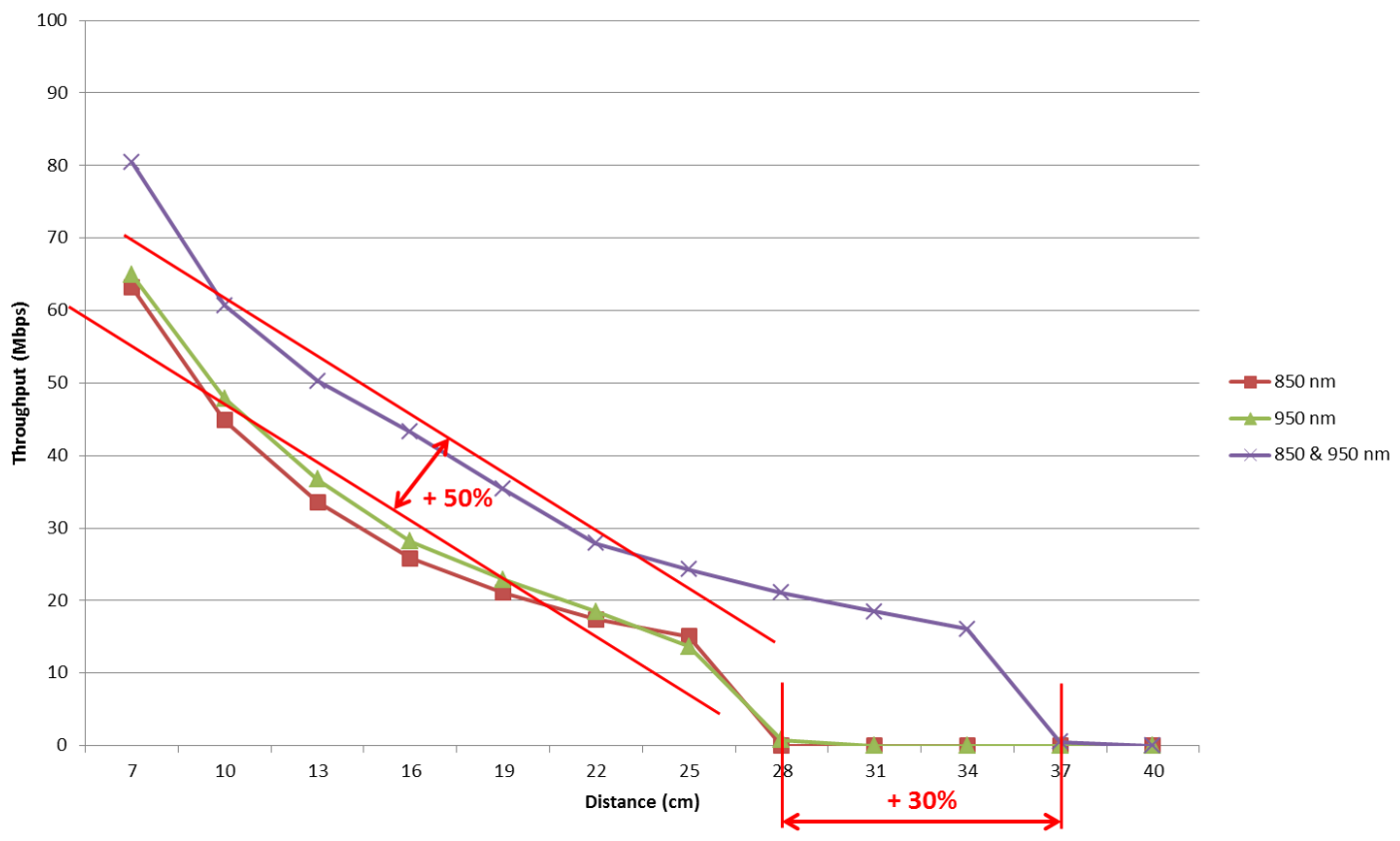

Figure 9. Throughput versus Distance

The experiment highlighted several points. First of all, it is possible to benefit from HPAV2 standard characteristics (TDD, MIMO, OFDM, CTC) in optical wireless communication device. It can be concluded that higher throughput and higher distance can be achieved by $850 \& 950$ association compared to the use of each wavelength separately. Additionally, data rate adaptation according to signal propagation modification offers robust link. The gains obtained are important, around $+50 \%$ in terms of throughput and $+30 \%$ for distance. A Front End Optic (FEO) modification by $\mathrm{TX}($ Laser)/RX(Avalanche PhotoDiode - APD) couple will offer a better performance both in terms of data rate and distance.

\section{Latency}

As for latency determination, the classical Ping command on DOS mode is used. The result has shown an answer under 1 millisecond for a round trip, on both directions.

\section{Long time testing}

Concerning the long time testing, the test duration is several hours to test the data transmission stability. This test is done for the transmission configurations and comfort zone tests conditions of the product. The results are:

- Long time testing: 24 hours,

- Distance: $0.14 \mathrm{~m}$

- Average downlink (DL) data rate: $23.22 \mathrm{Mbps}$,

- Average uplink (UL ) data rate: 19.29 Mbps,

- Error detected: no,

- Jitter: < 10ms.

These results are very positive and present link robustness. In addition, the events counter indicates no burst errors. 


\section{CONCLUSION AND OUTLOOK}

In this article, we presented Terabit (Tbps) wireless communication needs, with Virtual Reality (VR) application as part of WORTECS European collaborative project objectives. The proposed demonstrator, by the use of differential mode with two wavelengths, offers a significant gain in terms of throughput and distance; with a data rate adaptation. The result obtained is around $+50 \%$ for the throughput and $+30 \%$ for distance. It is possible to increase performance by using a TX(laser)/RX(APD PD) pair. The next step may be multi-user configuration testing with multiple prototypes despite 1.3 Gbps architecture network constraint. Other solutions are also envisaged because Optical Wireless Communication still offers a lot of potential [15].

\section{ACKNOWLEDGEMENTS}

This project has received funding from the European Union's Horizon 2020 research and innovation programme under grant agreement No 761329.

\section{REFERENCES}

[1] Frank Effenberger, "Industrial trends and roadmap of access", ECOC $42^{\text {nd }}$ European Conference on Optical Communication, September 18-22 2016.

[2] Ericsson Mobility Report, June 2018, https://www.ericsson.com/en/mobility-report/reports/june-2018.

[3] WORTECS H2020 - https://cordis.europa.eu/project/rcn/211056_en.html

[4] Marwan Badawi, Thomas Boggini, Jérome Royan and Christian Gallard, "Focus on Virtual Reality", WORTECS deliverable D2.3a, 2017, https://wortecs.cms.orange-labs.fr/.

[5] Olivier Bouchet, Micheline Perrufel, Suat Topsu and Hongyu Guan, " Acemind new indoor full duplex optical wireless communication prototype", Proceedings Volume 9979, Laser Communication and Propagation through the Atmosphere and Oceans V, SPIE Optical Engineering + Applications, 2016, San Diego, USA.

[6] Jian Song, Wenbo Ding, Fang Yang, Hui Yang, Bingyan Yu and Hongming Zhang, " An Indoor Broadband Broadcasting System Based on PLC and VLC", IEEE Transactions on Broadcasting, Volume 61 Issue 2, June 2015.

[7] Mohammad-Ali Khalighi, Yacine Jaafar, Fang Xu, Frédéric Chazalef and Salah Bourennane, " Double-laser differential signaling for suppressing background radiations in FSO systems", 25th Biennial Symposium on Communications, Proceedings of a meeting held 12-14 May 2010, Kingston, Ontario, Canada.

[8] Plug PLC AV1300 Gigabit. https://www.tp-link.com/fr/products/details/cat-18_TL-PA8015PKIT.html\#overview.

[9] LED $850 \mathrm{~nm}$ - SFH 4557 Osram. https:/www.osram.com/os/ecat/Radial\%20T1\%2034\%20SFH\%204557/com/en/class_pim_web_catalog_103489/global/prd_pim_device_2219781/

[10] LED $950 \mathrm{~nm}$ - SFH 4547 Osram. https:/www.osram.com/os/ecat/Radial\%20T1\%2034\%20SFH\%204557/com/en/class_pim_web_catalog_103489/global/prd_pim_device_2219781/

[11] PIN Photodiode - SFH 2500 Osram. https://www.osram.com/os/ecat/Radial\%20SMR\%20SFH\%202500\%20FA/com/en/class_pim_web_catalog_10 3489/global/prd_pim_device_2219618/

[12] Maxim amplifiers: https://www.maximintegrated.com/en/app-notes/index.mvp/id/3642

[13] Olivier Bouchet, "Wireless Optical Communication", Wiley, 2012.

[14] IXIA Performance assessment of network: https://www.ixiacom.com/fr/products/ixchariot

[15] Ton Koonen, "Optical Wireless Systems: Technology, Trends and Applications", Journal of Lightwave Technology ( Volume: 36, Issue: 8), April 15, 2018, Page(s): 1459 - 1467. 